\title{
Performance Assessment of Islanding Detection for Mul- ti-inverter Grid-connected Photovoltaic Systems
}

\author{
Xing Zhang, Dong Xie \\ College of Electric and Automation, Hefei University of Technology, Hefei, China \\ Email:XDY@tlu.edu.cn, xiedong1018@yahoo.com.cn
}

Received 2013

\begin{abstract}
Islanding detection is an essential function for safety and reliability in grid-connected Distributed Generation Systems (DGS). Passive and active islanding detection methods have been analyzed in literature considering DGS with only one inverter connected to the utility. With the big scale application of photovoltaic (PV) power systems, islanding detection technology of multi-inverter DGS has been paid more attention. This paper analyzes the performance of diverse islanding detection methods in multiple inverters grid-connected PV systems. Non-Detection Zones (NDZ) of multi-inverter systems in a load parameter space are used as analytical tool. The paper provides guidance for the islanding detection design in multiple grid-connected inverters.
\end{abstract}

Keywords: Photovoltaic Power Systems; Islanding Detection; Multi-inverter; Non-Detection Zones

\section{Introduction}

One problem for photovoltaic (PV) power system is islanding effect. When the grid is tripped for breakdown or overhaul, the PV system remains independent running status connected with the local load, this kind of phenomenon is called the islanding effect. This phenomenon will threaten the safety of power maintainer and power equipment. Therefore, the PV system must have islanding detection function to prevent the occurrence of islanding effect [1-2].

Two types of islanding detection methods (IDMs), the passive and active methods, have so far been developed. Among them, the active methods include Active Frequency Drifting (AFD) method, Active Frequency Drifting with Positive-Feedback (AFDPF) method, SlipMode Frequency Shifting (SMS) method and so on. Now the researches on IDM are mostly in single-inverter PV systems. With the development of economy and technology, multi-inverter PV systems will be used more extensively. So the researchers have paid more attention to the IDMs of multi-inverter systems. According to the analysis of non-detection zones (NDZs) in $\mathrm{Q}_{\mathrm{f}} \times \mathrm{f}_{0}$ frame[3], this paper investigates the performance of IDMs in multi- inverter PV systems, so as to provide a reference for the islanding detection design in multiple grid-connected inverters.

\section{Performance Analysis of Idms for Mul- ti-Inverter Operating in Parallel}

\subsection{Part of Inverters Adopt Passive IDMs}

The inverters in grid-connected PV systems usually use sinusoidal current control model based on the unit power factor [4]. So, in multi-inverter systems, the grid-connected inverters equipped with passive IDMs can be regard as a negative resistance which transport the active power to grid, the negative resistance together with the local load is equivalent to resistance $R_{\text {eq }}$, i.e.

$$
R_{e q}=\frac{R}{1-k}
$$

where $\mathrm{R}$ is the local load, $\mathrm{k}$ is the proportion of active power which is output by inverters equipped with passive IDMs relative to overall active power consumed by the local load. After the equivalent disposal, $\mathrm{R}_{\mathrm{eq}}$ 's quality factor $\mathrm{Q}_{\text {feq }}$ may be represented as:

$$
Q_{\text {feq }}=\omega_{0} R_{e q} C=\frac{Q_{f}}{1-k}
$$

where $\mathrm{Q}_{\mathrm{f}}$ is the original quality factor of the local load. It can be shown that $R_{\mathrm{eq}}$ 's quality factor equals to $1 /(1-\mathrm{k})$ times of $\mathrm{Q}_{\mathrm{f}}$. That is to say, if the inverters equipped with passive IDMs provide 50\% active power to local load (k $=0.5$ ), the equivalent quality factor $\mathrm{Q}_{\mathrm{feq}}$ equals to $2 \mathrm{Q}_{\mathrm{f}}$.Thus, if the single-inverter systems equipped with active IDMs can successfully detect the islanding when $\mathrm{Q}_{\mathrm{f}} \leq 2.5$, the multi-inverter systems can detect the islanding only if $\mathrm{Q}_{\mathrm{f}} \leq 1.25$, this means the NDZ has shifted towards the left. Obviously, this kind of situation in- 
creased the probability of islanding occurrence.

\subsection{Inverters Equipped with AFD and SMS IDMs}

Taking two grid-connected inverters for example, suppose that the proportion of active power for the local load provided by the inverter equipped with AFD IDM is $\mathrm{K}_{\mathrm{AFDpu}}$, and then the proportion of active power provided by the inverter equipped with SMS IDM is (1-K $\left.\mathrm{K}_{\mathrm{AFDpu}}\right)$. Therefore, the two inverter's current is

$$
\begin{gathered}
i_{A F D}=\sqrt{2} K_{A F D p u} I \sin \left(2 \pi f t+\theta_{A F D}\right) \\
i_{S M S}=\sqrt{2}\left(1-K_{A F D p u}\right) I \sin \left(2 \pi f t+\theta_{S M S}\right)
\end{gathered}
$$

where $\theta_{\text {AFD }}$ is the current initial phase angle of the inverter equipped with AFD IDM, and ${ }^{\theta}$ SFS is the current initial phase angle of the inverter equipped with SMS IDM[5-6]. As per definition:

$$
\theta_{A F D}=(2 \pi f) \cdot \frac{t_{Z}}{2}=\pi \cdot \frac{\Delta f}{f+\Delta f}
$$

where $t_{\mathrm{z}}$ is the dead area time inserted into the output current of inverter equipped with AFD IDM, $f$ is the last period frequency of inverter output voltage, $\Delta \mathrm{f}$ is the frequency shifting of inverter output voltage;

$$
\theta_{S M S}=\theta_{m} \cdot \sin \left(\frac{\pi}{2} \cdot \frac{f-f_{g}}{f_{m}-f_{g}}\right)
$$

where $\theta_{\mathrm{m}}$ is the maximum phase shifting of output current of inverter equipped with SMS IDM, $\mathrm{f}_{\mathrm{m}}$ is frequency when $\theta_{\mathrm{m}}$ arise, $\mathrm{f}_{\mathrm{g}}$ is the frequency of grid, $\mathrm{f}$ is the last period frequency of inverter output voltage.

According to (3) and (4), if the two parallel inverters are equivalent to one inverter, the initial phase angle of equivalent inverter's total output current is

$$
\theta_{I N V}=\tan ^{-1}\left(\frac{K_{A F D p u} \cdot \sin \theta_{A F D}+\left(1-K_{A F D p u}\right) \cdot \sin \theta_{S M S}}{K_{A F D p u} \cdot \cos \theta_{A F D}+\left(1-K_{A F D p u}\right) \cdot \cos \theta_{S M S}}\right)
$$

Thus, the NDZ in $\mathrm{Q}_{\mathrm{f}} \times \mathrm{f}_{0}$ frame is determined by the criteria as follows

$$
f^{2}+\frac{f_{0} \tan \left[\theta_{I N V}(f)\right]}{Q_{f}} f-f_{0}^{2}=0
$$

If $\Delta \mathrm{f}$ in AFD IDM is $1 \mathrm{~Hz}, \theta_{m}$ is $10^{\circ}$ and $\left(f_{\mathrm{m}}-f_{\mathrm{g}}\right)$ is $3 \mathrm{~Hz}$ in SMS IDM, the sketch map of NDZ for PV system with both AFD and SMS IDMs is shown in Figure 1.

There one sees that the NDZ is enlarged along with the augment of $\mathrm{K}_{\mathrm{AFDpu}}$, which proportion of local load's active power provided by the inverter equipped with AFD IDM.

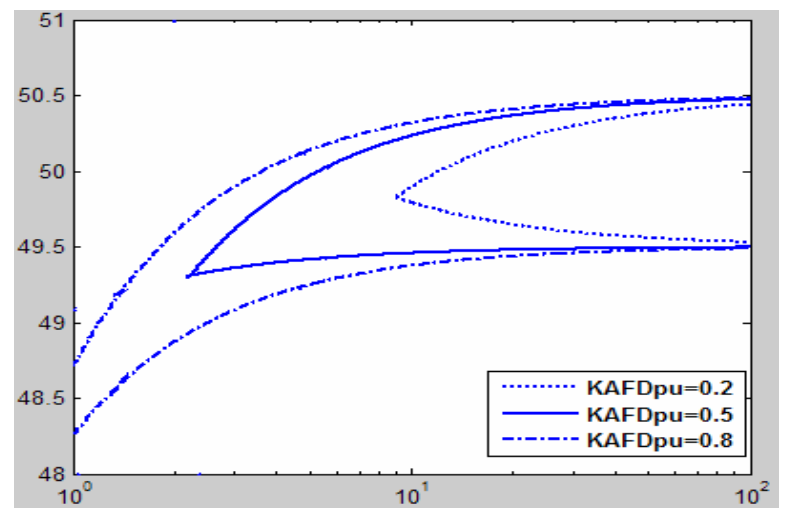

Figure 1. NDZ of a system with both AFD and SMS IDMs.

\subsection{Inverters equipped with AFD and AFDPF IDMs}

The AFD IDM shifts the system operating frequency towards the enhanced direction without considering if the local load is capacitor or inductor. But the AFDPF IDM will shift system operating frequency towards enhanced or descended direction, which is decided by local load's capacitive or inductive characteristic. Therefore, the multi-inverter PV systems equipped with both AFD IDM and AFDPF IDM, will have mutual influence.

Still taking two grid-connected inverters for example, suppose that the proportion of active power for the local load provided by the inverter equipped with AFD IDM is $K_{A F D p u}$, and then the proportion of active power provided by the inverter equipped with AFDPF IDM is $\left(1-K_{A F D p u}\right)$. Therefore the two inverter's current is [7]

$$
\begin{gathered}
i_{A F D}=\sqrt{2} K_{A F D p u} I \sin \left[2 \pi f t+\theta_{\mathrm{AFD}}\right] \\
i_{\mathrm{AFDPF}}=\sqrt{2}\left(1-K_{A F D p u}\right) I \sin \left(2 \pi f t+\theta_{\mathrm{AFDPF}}\right)
\end{gathered}
$$

where $\theta_{\mathrm{AFD}}$ is the current initial phase angle of the inverter equipped with AFD IDM, and $\theta_{\text {AFDPF }}$ is the current initial phase angle of the inverter equipped with AFDPF IDM. According to definition,

$$
\theta_{A F D P F}=(2 \pi f) \cdot \frac{t_{Z}}{2}=\frac{\pi}{2}\left(c f_{0}+k \Delta f\right)
$$

where $c f_{0}$ is the initial chopping factor, $\mathrm{k}$ is the positive-feedb-ack gain. The $\theta_{A F D}$ is the same as last section, thus the initial phase angle of equivalent inverter's total output current is:

$$
\theta_{I N V}=\tan ^{-1}\left(\frac{\tan ^{2} \alpha \cdot \sin \theta_{A F D}+\sin \theta_{A F D P F}}{\tan ^{2} \alpha \cdot \cos \theta_{A F D}+\cos \theta_{A F D P F}}\right)
$$

where $\alpha=\sin ^{-1}\left(\sqrt{K_{A F D p u}}\right)$. Similarly, as per (8), when $\Delta f=1 \mathrm{~Hz}, c f_{0}=0.03, k=0.06$, the sketch map of NDZ for PV system with both AFD and AFDPF IDMs can be shown in Figure 2. Figure 2 displays that the $\mathrm{NDZ}$ is enlarged along with the increase of $\mathrm{K}_{\mathrm{AFDpu}}$. It 


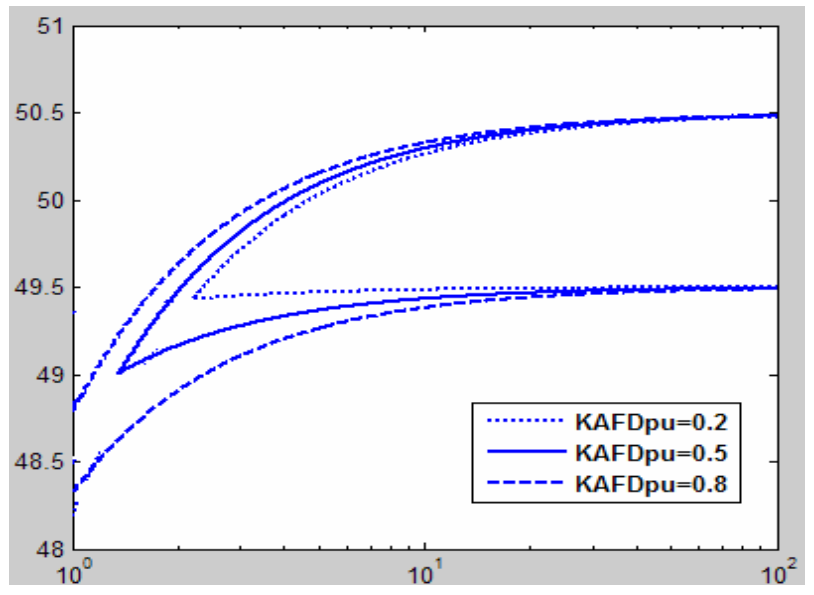

Figure 2. NDZ of a system with both AFD and AFDPF IDMs.

means that if local load augments the proportion of active power provided by the inverter equipped with AFD IDM, the NDZ will be enlarged[8].

\subsection{Inverters Equipped with both AFDPF IDMs}

There is measuring error when sensors measure the frequency of voltage, so the frequency measured in the two inverters supposed to be $f_{1}=f+\Delta f_{e}$ and $f_{2}=f-\Delta f_{e}$ respectively. When use the AFDPF IDMs, if the actual frequency $f$ more than reference frequency $f_{0}$, as a result of the positive-feedback, frequency $f$ will further rise, otherwise $f$ will drop. Therefore, the system operating frequency of these two inverters changes reversely because of the sensors measuring error. Thus, the islanding detection function of the two inverters disturbs mutually, namely has the so-called dilution effect, and the probability of islanding occurrence is enlarged. The impact of measuring error on NDZ is analyzed as follows [9].

In order to have a convenient analysis, the initial chopping $c f_{0}$ and positive-feedback gain $\mathrm{k}$ of the two inverters are supposed to be the same. First, the two inverters are equivalent to one inverter, the output current of equivalent inverter is:

$$
i_{I N V}=\sqrt{2} \frac{I}{2}\left[\sin \left(2 \pi f_{1} t+\theta_{A F D P F 1}\right)+\sin \left(2 \pi f_{2} t+\theta_{A F D P F 2}\right)\right]
$$

If the value of $\Delta f_{e}$ is small enough, we can obtain the approximate expression

$$
\begin{aligned}
i_{I N V} & \approx \sqrt{2} I \cos \left(\frac{\theta_{A F D P F 1}-\theta_{A F D P F 2}}{2}\right) \sin \left(2 \pi f t+\frac{\theta_{A F D P F 1}+\theta_{A F D P F 2}}{2}\right) \\
& \approx \sqrt{2} I_{I N V} \sin \left(2 \pi f t+\theta_{I N V}\right)
\end{aligned}
$$

where

$$
\theta_{I N V}=\frac{\theta_{A F D P F 1}+\theta_{A F D P F 2}}{2}=\frac{\pi}{2}\left(c f_{0}+k \Delta f\right)
$$

Compared with (15) and (11), two expressions are the same. It means that because of frequency measuring error, the positive-feedback perturbations produced by the two inverters counteract mutually, but the initial chopping and positive-feedback gain still exist, the frequency positive-feedback can still be triggered, the effect of islanding detection is not affected. Therefore, the NDZ for systems of multi-inverters equipped with AFDPF IDMs is approximately the same as the NDZ for systems of single-inverter equipped with identical IDMs. According to (8), the NDZ for systems of two inverters equipped with AFDPF IDMs is shown in Figure 3.

\subsection{Inverters Equipped with Both SMS IDMs}

First, still supposed the frequency of the two inverters has sensor measuring error, and the error is $+\Delta f_{e}$ and $-\Delta f_{e}$ respectively, i.e. the error amplitude is the same and error polarity is opposite. Then the output current of equivalent inverter is

$$
i_{I N V}=\sqrt{2} \frac{I}{2}\left[\sin \left(2 \pi f_{1} t+\theta_{S M S 1}\right)+\sin \left(2 \pi f_{2} t+\theta_{S M S 2}\right)\right]
$$

If the value of $\Delta f_{e}$ is small enough, we can obtain the approximate expression

$$
\begin{aligned}
i_{I N V} & \approx \sqrt{2} I \cos \left(\frac{\theta_{S M S 1}-\theta_{S M S 2}}{2}\right) \sin \left(2 \pi f t+\frac{\theta_{S M S 1}+\theta_{S M S 2}}{2}\right) \\
& \approx \sqrt{2} I_{I N V} \sin \left(2 \pi f t+\theta_{I N V}\right)
\end{aligned}
$$

where

$$
\begin{aligned}
& \theta_{I N V}=\theta_{m e q} \sin \left(\frac{\pi}{2} \cdot \frac{f-f_{g}}{f_{m}-f_{g}}\right) \\
& \theta_{\text {meq }}=\theta_{m} \cos \left(\frac{\pi}{2} \cdot \frac{\Delta f_{e}}{f_{m}-f_{g}}\right)
\end{aligned}
$$

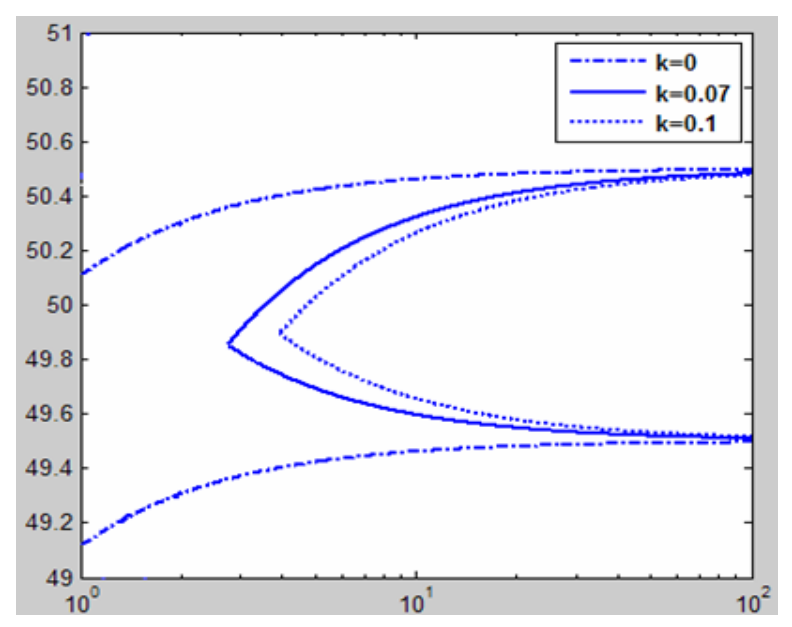

Figure 3. NDZ of a system with two AFDPF IDM inverters. 


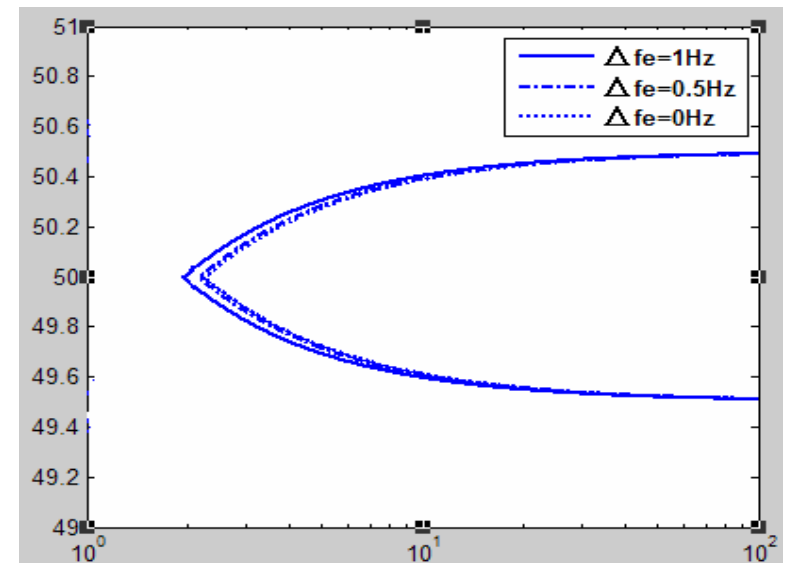

Figure 4. NDZ of a system with two SMS IDM inverters.

The (6) express the current initial phase angle of single-inverter system equipped with SMS IDMs. Compared with (19) and (6), we may see that the frequency measuring error will reduce the amplitude of maximum phase shifting angle $\theta_{m}$, it means that the positive-feedback perturbations produced by the two inverters counteract mutually, and the NDZ will increase, so the performance of islanding detection will drop. However, even if the measuring error $\Delta f_{e}$ takes a relatively big value $(0.5 \mathrm{~Hz})$, and $f_{\mathrm{m}}-f_{\mathrm{g}}=3 \mathrm{~Hz}$, the maximum phase shifting angle $\theta_{\text {meq }}$ drops only $3.4 \%$ compared with $\theta_{m}$, there isn't obvious change for the NDZ. Figure 4 shows the NDZ for different $\Delta f_{e}\left(\theta_{m}=10^{\circ}, f_{m}-f_{g}=3 \mathrm{~Hz}\right)$. This figure indicates that though the frequency measuring error makes the perturbation produced by the two inverters counteract mutually, it almost has no impact on performance of islanding detection ${ }^{[9]}$.

\section{Conclusions}

Synthesizing the above analysis, for the islanding detection performance of the multi-inverter grid-connected PV systems, we may draw the following conclusion:

First, when part of inverters in system adopt active IDMs, the others use passive IDMs, the application of passive IDMs enlarges the NDZ, and increases probability of islanding; Second, when part of inverters in system adopt AFD IDMs, the others use positive-feedback based active IDMs, such as SMS and AFDPF IDMs, the NDZ will be enlarged and hence increase the possibility of islanding if we magnify the proportion of load active power provided by inverters equipped with AFD IDM; Third, when all the inverters in system adopt AFDPF
IDMs or SMS IDMs, though the sensor measuring error makes the perturbation produced by the inverters counteract mutually, it almost has no impact on islanding detection performance even if in the worst situation(The inverters have identical error amplitude and opposite error polarity).

\section{REFERENCES}

[1] K. Agbossou, M. Kolhe, J. Hamelin and T. K. Bose, "Performance of a Stand-Alone Renewable Energy System Based on Energy Storage as Hydrogen," IEEE Transactions on Energy Conversion, Vol.19, No. 3, 2004, pp. 633-640. doi.org/10.1109/TEC.2004.827719

[2] IEEE Std.929-2000, "IEEE Recommended Practice for Utility Interface of Photovoltaic (PV) System," IEEE Std.929-2000, Apr.2000.

[3] Z. Ye, A. Kowalkar, Y. Zhang, P. Du and R. Walling, "Evaluation of Anti-islanding Schemes based on non Detection Zone Concept," IEEE Trans on Power Electron, Vol. 19, No. 5, 2004, pp. 1171-1176.

doi.org/10.1109/TPEL.2004.833436

[4] S. J. Huang and F. S. Pai, "Design and Operation of Grid-connected Photovoltaic System with Power-factor Control and Active Islanding Detection," IEEE Proceedings on Generation, Transmission and distribution, Vol. 148, No. 2, 2001, pp. 243-250.

[5] A. Cardenas, Agbossou and M. L. Doumbia, "An Active Anti-Islanding Algorithm for Inverter Based Multi-Source DER Systems," IEEE Asia-Pacific Power and Energy Engineering Conference, APPEEC 2009, 27-31 March, 2009, pp. 1-6.

[6] F. R. Liu, Y. Kang and S. X. Duan, "Analysis and Optimization of Active Frequency Drift Islanding Detection Method," in Twenty Second Annual IEEE Applied Power Electronics Confer., APEC 2007, pp. 379-1384.

[7] L. A. C. Lopes and H. Sun, "Performance Assessment of Active Frequency Drifting Islanding Detection Methods," IEEE Transactions on energy Conversion, Vol. 21, No. 1, 2006, pp. 171-180. doi:10.1109/TEC.2005.859981

[8] M. L. Doumbia, K. Agbossou and D. Tran-Khanh-Viet, "Correlation Technique Investigation for Islanding Detection of Inverter based Distributed Generation," in IEEE Power Electronics Specialists Conference-PESC2008, 2008, pp. 4556-4561.

[9] A. Cardenas, K. Agbossou and M. L. Doumbia, "Islanding Detection Method for Multi-Inverter Distributed Generation," Journal of Electromagnetic Analysis and Applications, Vol. 1 No. 3, 2009, pp. 170-180.

doi:10.4236/jemaa.2009.13026 